\title{
Problemas de sono-vigília em crianças: um estudo da prevalência
}

\author{
John M. Klein - Universidade de Trier \\ Alda Gonçalves - Universidade do Minho
}

\begin{abstract}
Resumo
A investigação recente tem demonstrado que os problemas de sono estão entre as queixas mais comuns ao longo de toda a infância. Alterações nos padrões de sono têm efeitos nefastos no funcionamento neuropsicológico, que se reflecte no bem-estar psicológico das crianças. O presente estudo procurou avaliar os hábitos relacionados com o sono e estimar a prevalência de problemas do sono em crianças que frequentam o $1^{\circ}$ Ciclo do Ensino Básico, nas escolas dos concelhos de Braga e Faro. Para isso, foi aplicado o Questionário de Experiências de Sono-Vigília a uma amostra composta por 938 crianças, das quais 485 (52\%) do sexo feminino, com idades compreendidas entre os 7 e os 11 anos de idade $(M=8,74 \pm D P=0,989)$. Verificou-se que $56,4 \%$ das crianças dormem mais de nove horas por noite e 18,6\% das crianças apresentam perturbações do sono. Os resultados serão discutidos em termos da relação entre a presença de perturbação do sono na infância e o desenvolvimento de psicopatologia na idade adulta. Por fim, será discutida a importância deste questionário para estudo das problemáticas associadas ao sono e a contribuição destes dados epidemiológicos para a investigação e prática clínica no nosso país, no sentido de sensibilizar os técnicos e os agentes educativos para a existência desta problemática.

Palavras-chave: Sono; Epidemiologia; Crianças em idade escolar.
\end{abstract}

\section{Sleep-wake problems in children: A study about prevalence}

\begin{abstract}
Recent studies demonstrated that sleep disturbances are among the most common complaints throughout childhood. Changes in the sleep patterns have negative effects in the neuropsychological functioning, who affects the children's mental well being. The present paper aims to evaluate the sleep habits and estimate the prevalence of sleep disturbances in the Portuguese school-aged children of two regions: Faro and Braga. A total of 938 elementary school students, of which 485 were girls $(52 \%)$, aged between 7 and 11 years $(M=8,74 \pm S D=0,989)$ were used for this study. The results indicate that $56,4 \%$ of the children's sleeps more than 9 hors during the night and; $18,6 \%$ has sleep disturbances. The results will be discussed regarding the linkage between the presence of sleep psychopathology in infancy and the development of psychopathology in adulthood. Further the importance of this questionnaire for sleep related problems studies so as the epidemiological data gathered with it will be discussed in terms of their relevance for research and clinical practice in Portugal, in order to acknowledge the clinicians and educational agents for such problems.
\end{abstract}

Keywords: Sleep; Epidemiology; School-aged children.

\section{Introdução}

As perturbações do sono estão entre as queixas mais comuns ao longo de toda a infância. De acordo com os registos de pediatras, estas estão em quinto lugar no que respeita às preocupações dos pais; a seguir as doenças físicas, alimentação, problemas comportamentais e deficiências físicas (Mindell, Boaz, Joffe, Curtis \& Birley, 2004). Nos últimos anos, a comunidade científica tem vindo a atribuir cada vez maior relevância ao estudo da qualidade dos padrões de sono no período da infância. Sendo este considerado um importante factor não só ao nível do desenvolvimento das aprendizagens escolares, como de bem-estar. Os problemas de sono nas crianças têm sido considerados transitórios, sem gravidade e sem consequências a longo prazo. Não muitas vezes, estes problemas têm sido indevidamente menosprezados e remetidos para segundo plano, podendo frequentemente progredir para estados mais severos e afectar o desenvolvimento e bem-estar das crianças (Chervin, Rillon, Basseti, Ganoczy \& Pituch, 1997; Mindell, Moline, Zendell, Brown \& Frey, 1994; Stores, 1996).

Os primeiros estudos epidemiológicos relativos aos hábitos de sono das crianças foram realizados no início do séc. XX. Das primeiras avaliações destaca-se um estudo efectuado na Califórnia (Terman \& Hocking, 1913), no qual os investigadores analisaram os horários de 2.692 crianças e concluíram que nas crianças em idade escolar a

${ }^{1}$ Endereço para correspondência:

Klinisch Psychologie und Psychotherapie, Fachbereich I - Psichologie

Universität Trier, D - 54.286 - Trier - Deutschland

E-mail: john.klien@uni-trier.de 
duração do período de sono era superior a 11 horas. Estudos mais recentes têm mostrado que aproximadamente um terço de todas as crianças sofre de problemas do sono (Blader \& Gallagher, 2001; Kahn e cols., 1989; Rona, Guilford \& Chinun, 1998; Simonds \& Parraga, 1984), os quais são recorrentes, estimando-se a sua prevalência entre 17\% e 41\% (Archobold, Pituch, Panahi \& Chervin, 2002; Manni e cols., 1997; Pollock, 1994). Diferentes problemas de sono são característicos de diferentes idades, no entanto a insónia parece ser a perturbação mais comum em todas as faixas etárias (Wiggs \& Stores, 2001). As crianças mais jovens apresentam tipicamente perturbações de sono de cariz mais comportamental, enquanto nas crianças em idade escolar predominam diferentes formas de insónia latência prolongada do sono, acordar durante a noite ou acordar muito cedo (Blader, Koplewicz, Abikoff \& Foley, 1997; Kahn e cols., 1989). No entanto, e apesar destes dados algo alarmantes, apenas um número restrito de estudos tem demonstrado que estes problemas são persistentes. É necessário um maior número de estudos longitudinais de modo a esclarecer as relações causais subjacentes e os sintomas psiquiátricos associados (Dahl, 1989; Klackenberg, 1982; Zuckerman, Stevenson \& Bailey, 1987).

Em Portugal, a quantidade de estudos existentes na literatura no âmbito da temática das perturbações do sono é consideravelmente menor quando comparada com o panorama internacional, sendo que, actualmente, apenas são conhecidos dois estudos cuja população-alvo foram crianças em idade escolar. O estudo de Clemente, Silva, Ferreira, César e Azevedo (1997), realizado com 988 crianças do $1^{\circ}$ Ciclo do Ensino Básico (CEB) provenientes de escolas de Coimbra, procurou caracterizar várias dimensões: a) estudar os hábitos de sono, b) estimar a relação entre comportamentos do sono e variáveis demográficas, c) medir a associação entre comportamentos do sono, d) estimar a relação entre comportamentos do sono e psicopatologia, e e) estimar a relação entre comportamentos do sono e rendimento escolar. Este estudo demonstrou que algumas crianças apresentavam hábitos de sono desadequados, sem que no entanto houvesse uma relação significativa entre problemas de sono e psicopatologia, e problemas de sono e rendimento escolar. Num outro estudo, realizado no contexto do Serviço de Pediatria do Hospital de Santo António no Porto, foram entrevistados pais de crianças dos 6 aos 10 anos, com o objectivo de conhecer alguns hábitos e distúrbios do sono da população infantil nesta faixa etária (Borges, Azevedo, Fonseca, Torres \& Costa, 2000). Dos resultados encontrados, salienta-se que $98 \%$ dormiam mais de 8 horas, $24 \%$ acordavam durante a noite, $42 \%$ utilizavam ou tinham utilizado um objecto de transição, 47\% necessitavam de outras condições para adormecer e em 51\% havia ocorrência de eventos durante o sono. Foi também encontrada uma correlação, com significado estatístico, dos eventos do sono com: primeiros filhos, "cólicas do lactente", história familiar de problemas de sono, utilização prévia ou actual de objecto de transição, necessidade de outras condições para adormecer, e alterações de comportamento e patologias associadas.

Deste modo, a epidemiologia das perturbações do sono ainda se encontra a dar os seus primeiros passos. A sua juvenilidade está patente na ausência de uma definição consensual relativamente ao conjunto das síndromes do sono e como estas deverão ser avaliadas na população, o que evidencia a sua importância ao nível clínico e epidemiológico (Ohayhon, 2002). Com base nestes estudos e face à escassez de dados relativos à problemática do sono na infância no panorama português, a presente investigação procura avaliar os hábitos relacionados com o sono e estimar a prevalência de problemas do sono, em crianças que frequentam o $1^{\circ}$ Ciclo do Ensino Básico, em escolas das cidades de Braga e Faro.

\section{Método}

\section{Amostra}

A amostra do presente estudo foi obtida mediante um método de amostragem polietápica, com recurso a amostragem aleatória em cada uma das fases. As diferentes etapas consistiram em seleccionar dois distritos de todo o país, seguidos das suas capitais de distrito. Deste modo, os dados foram recolhidos, no ano lectivo de 2004/2005, em duas cidades de Portugal Continental, nomeadamente Faro (50,4\%) e Braga $(49,6 \%)$, sendo os dados relativos aos últimos três meses anteriores à aplicação do questionário, em pleno período lectivo. De forma a garantir uma elevada representatividade da população-alvo, procurou-se abraçar $1 / 6$ da mesma. Para tal, e inerente ao processo de amostragem, foi realizado um levantamento da população do $1^{\mathrm{o}}$ CEB compreendida entre as $2^{\mathrm{a}}$ e $4^{\mathrm{a}}$ classes.

\section{Instrumento}

O Questionário de Experiências de SonoVigília (QESV; Diest, 1990) é uma medida de autorelato padronizada de avaliação do sono-vigília ao longo de um ciclo de 24 horas. É constituído por 14 itens e compreende 8 domínios, que correspondem a diferentes tipos de queixas do sono, durante um período de três meses: insónia inicial (itens 1 e 2), 
insónia de manutenção (itens 3 e 4), insónia terminal (itens 5 e 6), sonolência ao acordar (itens 7 e 8), cansaço ao acordar (itens 9 e 10) e sonolência diurna (item 11 e 13); sendo que os restantes dois domínios correspondem a aspectos que podem influenciar o sono, tais como: ressonar (item 12) e a duração diária do sono (item 14). Os cinco primeiros domínios já referenciados são constituídos por dois itens: um item específico, que permite avaliar a presença de uma determinada queixa, e um item de frequência que permite avaliar a frequência com que essa queixa ocorre. Em relação à sonolência diurna, este domínio é avaliado a partir da frequência de dois descritores (sestas e dificuldade em manter-se desperto durante o dia). Todos os itens têm quatro categorias de resposta, que vão desde o de modo nenhum a muito grave ou sempre. Cada item é ponderado numa escala de Likert de quatro pontos, e cada uma das respostas é cotada de 1 a 4. A pontuação de cada uma das queixas constituídas por dois itens é o valor do produto das cotações e situa-se entre 1 e 16. A soma das pontuações, que pode variar entre 6 e 96, dá-nos o valor total de perturbação do sono.

Neste estudo, foi administrada a versão portuguesa do QESV (Klein, Fernandes \& Gonçalves, 2005), à qual foi anexada uma ficha de dados sóciodemográficos que procurava caracterizar aspectos relativos à idade, género, escolaridade e localidade. Este questionário está validado para a população portuguesa numa faixa etária dos 6 aos 11 anos, tendo revelado uma adequada fidelidade $(a=0,73)$ e validade (Klein, Silva \& Gonçalves, no prelo).

\section{Procedimento}

Primeiramente, foram solicitadas as respectivas autorizações às Direcções Regionais do Norte e do Algarve, onde juntamente foram anexadas as referentes considerações relativas aos procedimentos éticos a ter em conta nesta investigação. Após o devido consentimento, foram estabelecidos os contactos com as escolas e respectivos professores, de forma a elaborar um plano de recolha dos dados que não incomodasse as actividades na sala de aula.

Seguidamente, os pais foram informados por carta dos objectivos da investigação e foi-lhes solicitada autorização para a recolha de dados junto dos seus filhos; uma vez recebida a respectiva autorização dos pais, a cada criança, individualmente, foram-lhe verbalmente esclare- cidos os objectivos, confidencialidade e dinâmica do processo de recolha de dados, pelos investigadores.

Numa terceira fase, os professores receberam treino na aplicação do instrumento e na sintomatologia do sono nele incluída, de modo a lidarem da forma mais adequada com dúvidas que, tendo em vista a faixa etária em estudo, pudessem surgir. Mais ainda, um investigador estava sempre contactável e disponível caso o professor tivesse alguma indecisão no que concerne à aplicação e/ou ao preenchimento do questionário.

Os dados foram recolhidos com recurso a um processo especial administrado pelo professor da turma. Este atribuía um código numérico a cada estudante da sua sala de aula, com o qual assinalava o questionário do respectivo aluno. Os professores administraram o questionário num horário flexível, numa semana previamente combinada com os investigadores, mas não logo nos primeiros minutos da aula ou após um intervalo. Os alunos levaram cerca de 15 minutos a preencher o questionário e, uma vez esta tarefa terminada, os questionários foram colocados num envelope e levados para a secretaria da escola, onde foram recolhidos pelos investigadores.

\section{Resultados}

Os resultados foram analisados com recurso ao SPSS 12.0 (2004). Foram utilizadas estatísticas nãoparamétricas (correlações de Spearman, teste de MannWhitney) para determinar possíveis relações e diferenças entre os problemas de sono e o género e/ou os aspectos demográficos. Em primeiro lugar serão apresentados os resultados referentes às características sócio-demográficas e à duração de sono das crianças; de seguida serão expostos os dados relativos ao score total do QESV (isto é, a perturbação de sono em geral) e dos sintomas-chave. Um nível de significância de $p=0,05$ foi considerado a priori como definição de significância estatística.

\section{Características sócio-demográficas da amostra}

De um total de 1.028 crianças que foram inquiridas para este estudo, 58 (5,64\%) foram excluídas por não terem completado o questionário e 32 (3,11\%) por apresentarem problemas e/ou diagnósticos médicopsiquiátricos. Assim, a amostra é composta por 938 (91,25\%) crianças, das quais 488 (52\%) do sexo feminino e $450(48 \%)$ do sexo masculino, com idades compreendidas entre os 7 e os 11 anos de idade $(M=8,74 \pm D P=0,989)$, que frequentam o $1^{\circ}$ Ciclo do Ensino Básico. 


\section{Duração do sono das crianças ( $N=938$ )}

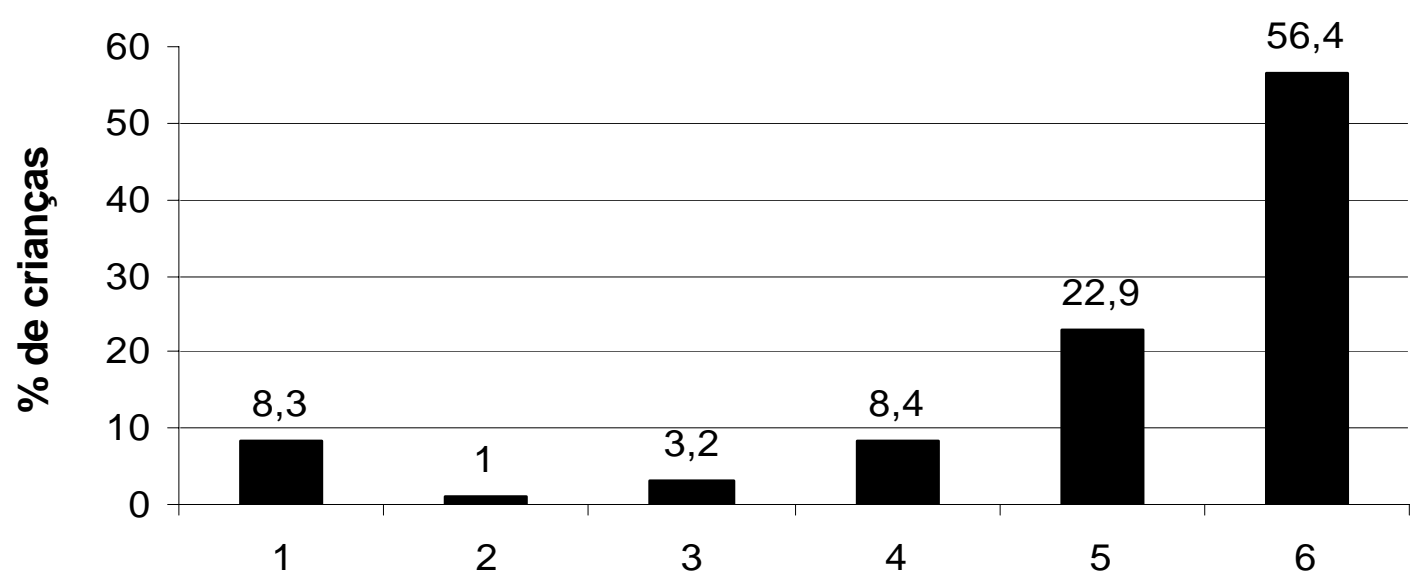

Grupos: $1=<5 h ; 2=<6 h ; 3=<7 h ; 4=<8 h ; 5=<9 h ; 6=>9 h$

Figura 1 - Duração média do sono por noite (horas)

Tal como se pode observar na Figura 1, há uma considerável proporção de crianças com duração média do sono de mais de 9 horas por noite. Porém, verifica-se a existência de um significativo número de crianças que dorme menos de 5 horas por noite. Para mais, as crianças do concelho de Faro $(M=5,17 ; \quad D P=1,38)$ dormem significativamente mais que as crianças do concelho de Braga $(M=4,95 ; D P=1,55 ; Z=-2,449 ; p=0,014)$. Em termos de possíveis diferenças de género, não se constataram diferenças estatisticamente significativas, entre os rapazes $(M=4,95 ; D P=1,53)$ e as raparigas $(M=5,16 ; D P=1,40)$, relativamente à duração do sono $(Z=-1,854: p=0,064)$. Não se verificou uma relação entre a duração do sono e a qualidade de sono - nota total do QESV (rhố $=0,054$, $n=938$ ).

\section{Perturbação do sono}

No que concerne ao valor total da perturbação do sono, $18,6 \%(n=174)$ das crianças apresentaram queixas significativas, sendo que $13,1 \%(n=123)$ são de grau leve, $4,2 \% \quad(n=39)$ preenchem os critérios de diagnóstico dos sintomas e 1,1\% $(n=10)$ manifestam sintomas com elevada gravidade. Não foram encontradas diferenças estatisticamente significativas em relação ao género e à localização geográfica.

\section{Insónia inicial}

Relativamente ao sintoma insónia inicial, 29,4\% $(n=276)$ das crianças apresentaram queixas significativas, sendo 20,3\% $\quad(n=190) \quad$ de grau leve, 8,5\% $\quad(n=80)$ preenchendo o critério de diagnóstico do sintoma e $0,6 \%$ $(n=6)$ manifestando elevada gravidade no sintoma. Verificou-se que as crianças de Faro $(M=3,71 ; D P=2,71)$ apresentam scores significativamente superiores aos das crianças de Braga $(M=3,28 ; D P=2,67 ; Z=-4,856 ; p=0,000)$. No que se refere ao género, as crianças do sexo masculino $(M=3,81 ; D P=2,98)$ exibem valores superiores às crianças do sexo feminino $(M=3,21 ; D P=2,37 ; Z=-2,654 ; p=0,000)$.

\section{Insónia de manutenção}

Referente à problemática insónia de manutenção, $31,9 \% \quad(n=299)$ das crianças apresentam-na, das quais $17,5 \% \quad(n=164)$ apresentaram queixas ligeiras, $13,1 \%$ $(n=123)$ preencheram o critério de diagnóstico para a presença do sintoma e 1,3\% revela elevada gravidade face ao sintoma. As crianças de Braga demonstraram valores mais elevados $(M=4,02 ; D P=3,41)$ em relação às de Faro $(M=3,37 ; D P=2,92 ; Z=-2,440 ; p=0,015)$.

\section{Insónia terminal}

No que concerne ao sintoma Insónia terminal, $35,9 \% \quad(n=337) \quad$ das crianças apresentaram queixas significativas; em que, $14,3 \%(n=134)$ são de grau leve, $20,5 \%(n=192)$ preencheram o critério de diagnóstico para a presença do sintoma e $1,1 \%(n=10)$ apresentaram elevada gravidade relativamente a este sintoma. Verificou-se que as crianças do conselho de Braga $(M=4,94 ; \quad D P=3,11)$ mostram valores superiores em relação às crianças de Faro $(M=4,55 ; \quad D P=2,94)$, face a este sintoma $(Z=-2,514$; $p=0,012)$.

\section{Sonolência ao acordar}

Em relação ao indicativo sonolência ao acordar, 26,6\% $(n=250)$ das crianças exibiram queixas significativas., sendo $11,6 \%(n=109)$ sintomatologia leve, 13,8\% $(n=129)$ satisfazendo o critério de diagnóstico para a presença do Psico-USF, v. 13, n. 1, p. 51-58, jan./jun. 2008 
sintoma e $1,2 \%(n=11)$ mostrando elevada gravidade neste sintoma. Verificou-se que as crianças de Braga $(M=3,86$; $D P=3,06)$ apresentam resultados mais elevados face às crianças de Faro $(M=3,78 ; D P=2,95 ; Z=-2,859 ; p=0,004)$.

\section{Cansaço ao acordar}

No que se refere ao indicativo cansaço ao acordar, $11,9 \% \quad(n=112) \quad$ das crianças mostraram queixas significativas; onde 6,4\% $(n=60)$ são de grau leve, $4,8 \%$ $(n=45)$ perfazem o critério de diagnóstico para a presença do sintoma e $0,7 \%(n=6)$ apresenta elevada gravidade no respectivo sintoma. Não foram encontradas diferenças estatisticamente significativas em relação ao género e à localização geográfica.

\section{Sonolência diurna}

No que respeita ao sintoma sonolência diurna, $9,2 \%(n=86)$ das crianças revelaram queixas significativas; onde 1,4\% $(n=13)$ é sintomatologia leve, 6,2\% $(n=58)$ satisfazem o critério de diagnóstico para a presença do sintoma e $1,6 \%(n=15)$ apresenta elevada gravidade face ao sintoma. Verificou-se que as crianças do sexo masculino $(M=2,93 ; D P=3,29)$ apresentam valores mais elevados que as crianças do sexo feminino $(M=2,05 ; D P=2,57 ; Z=-$ $2,654 ; p=0,008)$.

\section{Ressonar}

Verificou-se, no que concerne ao sintoma ressonar (avaliado pelo item 12), 17,9\% $(\mathrm{n}=168)$ das crianças referem-no como ocorrendo poucas vezes, 6,1\% $(n=57)$ muitas vezes e $4,1 \%(n=38)$ sempre. Constatou-se ainda que as crianças de Faro $(M=1,51 ; D P=0,83)$ relataram maior frequência desta queixa que as de Braga $(M=1,34$; $D P=0,72 ; Z=-3,326 ; p=0,001)$.

\section{Relação entre a idade e sintomatologia do sono}

Verificou-se uma correlação negativa baixa entre a idade e os seguintes sintomas: insónia inicial $(r h \hat{o}=-0,130$; $p=<0,000)$; insónia de manutenção ( $r h \hat{o}=-0,136 ; p=<0,000)$; insónia terminal $(r h \hat{o}=-0,111 ; p=<0,000)$ e cansaço ao acordar $(r h \hat{o}=-0,091 ; p=<0,000)$.

\section{Prevalência de sintomatologia do sono por idade}

Conforme se pode constatar na Tabela 1, que apresenta o número de crianças e a distribuição da prevalência do sintoma (e/ou perturbação do sono) em função da idade; as idades mais afectadas pelos problemas de sono são as de crianças com 8 e 9 anos. No que se refere à idade dos 8 anos, verifica-se que 35 (40,7\%) crianças apresentam insónia inicial, 41 (31,8\%) manifestam insónia de manutenção, $61(31,0 \%)$ têm insónia terminal, $40(28,6 \%)$ exibem sonolência ao acordar, 12 (24,5\%) apresentam cansaço ao acordar, 25 (36,8\%) manifestam sonolência diurna; havendo um total de 232 crianças desta idade que possuem perturbação do sono, ou seja 30,8\% das crianças com perturbação do sono têm 8 anos de idade.

No que se refere à idade dos 9 anos, verifica-se que $28(32,6 \%)$ crianças apresentam insónia inicial, 42 (32,6\%) manifestam insónia de manutenção, 61 (31,3\%) têm insónia terminal, $61(43,6 \%)$ exibem sonolência ao acordar, 31 (63,3\%) apresentam cansaço ao acordar, 31 (45,6\%) manifestam sonolência diurna; sendo o total de crianças de 9 anos com perturbação do sono de 317 (42,0\%).

Tabela 1 - Distribuição da sintomatologia de perturbação do sono pela idade

\begin{tabular}{|c|c|c|c|c|c|c|c|c|c|c|}
\hline \multicolumn{11}{|c|}{ Idade } \\
\hline \multirow[t]{2}{*}{ Sintomas } & \multicolumn{2}{|c|}{7} & \multicolumn{2}{|c|}{8} & \multicolumn{2}{|c|}{9} & \multicolumn{2}{|c|}{10} & \multicolumn{2}{|c|}{11} \\
\hline & $\begin{array}{c}\text { SS } \\
\mathrm{n}(\%)\end{array}$ & $\begin{array}{c}\text { CS } \\
\mathrm{n}(\%)\end{array}$ & $\begin{array}{c}\text { SS } \\
\mathrm{n}(\%)\end{array}$ & $\begin{array}{c}\text { CS } \\
\mathrm{n}(\%)\end{array}$ & $\begin{array}{c}\text { SS } \\
\mathrm{n}(\%)\end{array}$ & $\begin{array}{c}\text { CS } \\
\mathrm{n}(\%)\end{array}$ & $\begin{array}{c}\text { SS } \\
\mathrm{n}(\%)\end{array}$ & $\begin{array}{c}\text { CS } \\
\mathrm{n}(\%)\end{array}$ & $\begin{array}{c}\text { SS } \\
\mathrm{n}(\%)\end{array}$ & $\begin{array}{c}\text { CS } \\
\mathrm{n}(\%)\end{array}$ \\
\hline Insónia inicial & $\begin{array}{c}69 \\
(8,1 \%)\end{array}$ & $3(3,5 \%)$ & $\begin{array}{c}282 \\
(33,3 \%)\end{array}$ & $\begin{array}{c}35 \\
(40,7 \%)\end{array}$ & $\begin{array}{c}352 \\
(41,4 \%)\end{array}$ & $\begin{array}{c}28 \\
(32,6 \%)\end{array}$ & $\begin{array}{c}122 \\
(14,4 \%)\end{array}$ & $6(7,0 \%)$ & $\begin{array}{c}24 \\
(2,8 \%)\end{array}$ & $\begin{array}{c}14 \\
(16,3 \%)\end{array}$ \\
\hline $\begin{array}{l}\text { Insónia de } \\
\text { manutenção }\end{array}$ & $\begin{array}{c}59 \\
(7,7 \%)\end{array}$ & $\begin{array}{c}10 \\
(7,8 \%)\end{array}$ & $\begin{array}{c}251 \\
(32,9 \%)\end{array}$ & $\begin{array}{c}41 \\
(31,8 \%)\end{array}$ & $\begin{array}{c}324 \\
(42,4 \%)\end{array}$ & $\begin{array}{c}42 \\
(32,6 \%)\end{array}$ & $\begin{array}{c}106 \\
(13,9 \%)\end{array}$ & $\begin{array}{c}22 \\
(17,1 \%)\end{array}$ & $\begin{array}{c}24 \\
(3,1 \%)\end{array}$ & $\begin{array}{c}14 \\
(10,9 \%)\end{array}$ \\
\hline $\begin{array}{l}\text { Insónia } \\
\text { terminal }\end{array}$ & $\begin{array}{c}61 \\
(8,6 \%)\end{array}$ & $\begin{array}{c}11 \\
(5,6 \%)\end{array}$ & $\begin{array}{c}209 \\
(29,6 \%)\end{array}$ & $61(31, \%)$ & $\begin{array}{c}299 \\
(42,3 \%)\end{array}$ & $\begin{array}{c}61 \\
(31,3 \%)\end{array}$ & $\begin{array}{c}117 \\
(16,5 \%)\end{array}$ & $11(5,6 \%)$ & $\begin{array}{c}21 \\
(3,0 \%)\end{array}$ & $16(8,2 \%)$ \\
\hline $\begin{array}{l}\text { Sonolência ao } \\
\text { acordar }\end{array}$ & $\begin{array}{c}66 \\
(8,3 \%)\end{array}$ & $5(3,6 \%)$ & $\begin{array}{c}278 \\
(35,1 \%)\end{array}$ & $\begin{array}{c}40 \\
(28,6 \%)\end{array}$ & $\begin{array}{c}318 \\
(40,1 \%)\end{array}$ & $\begin{array}{c}61 \\
(43,6 \%)\end{array}$ & $\begin{array}{c}120 \\
(15,1 \%)\end{array}$ & $7(5,0 \%)$ & $\begin{array}{c}11 \\
(1,4 \%)\end{array}$ & $\begin{array}{c}27 \\
(19,3 \%)\end{array}$ \\
\hline $\begin{array}{l}\text { Cansaço ao } \\
\text { acordar }\end{array}$ & $\begin{array}{c}68 \\
(8,0 \%)\end{array}$ & $3(6,1 \%)$ & $\begin{array}{c}280 \\
(32,9 \%)\end{array}$ & $\begin{array}{c}12 \\
(24,5 \%)\end{array}$ & $\begin{array}{c}342 \\
(40,1 \%)\end{array}$ & $\begin{array}{c}31 \\
(63,3 \%)\end{array}$ & $\begin{array}{c}126 \\
(14,8 \%)\end{array}$ & $2(4,1 \%)$ & $\begin{array}{c}36 \\
(4,2 \%)\end{array}$ & $1(2,0 \%)$ \\
\hline $\begin{array}{l}\text { Sonolência } \\
\text { diurna }\end{array}$ & $\begin{array}{c}69 \\
(8,7 \%)\end{array}$ & $3(4,4 \%)$ & $\begin{array}{c}262 \\
(32,9 \%)\end{array}$ & $\begin{array}{c}25 \\
(36,8 \%)\end{array}$ & $\begin{array}{c}328 \\
(41,2 \%)\end{array}$ & $\begin{array}{c}31 \\
(45,6 \%)\end{array}$ & $\begin{array}{c}113 \\
(14,2 \%)\end{array}$ & $\begin{array}{c}9 \\
(13,2 \%)\end{array}$ & $\begin{array}{c}25 \\
(3,1 \%)\end{array}$ & $\begin{array}{c}0 \\
(0 \%)\end{array}$ \\
\hline $\begin{array}{l}\text { Perturbação de } \\
\text { sono }\end{array}$ & $\begin{array}{c}66 \\
(8,8 \%)\end{array}$ & $2(8,4 \%)$ & $\begin{array}{c}232 \\
(30,8 \%)\end{array}$ & $\begin{array}{c}232 \\
(30,8 \%)\end{array}$ & $\begin{array}{c}27 \\
(50,9 \%)\end{array}$ & $\begin{array}{c}317 \\
(42,0 \%)\end{array}$ & $\begin{array}{c}116 \\
(15,4 \%)\end{array}$ & $6(11,3 \%)$ & $\begin{array}{c}23 \\
(3,1 \%)\end{array}$ & $1(1,9 \%)$ \\
\hline
\end{tabular}

SS - Sem sintomatologia clinicamente significativa;

CS - Com sintomatologia clinicamente significativa. 


\section{Discussão}

O presente estudo permite afirmar que as perturbações de sono não são entidades nosológicas raras em crianças dos 7 aos 11 anos no nosso país. Cerca de $19 \%$ das crianças apresentaram problemas relacionados com o sono, sendo a insónia de manutenção, a insónia terminal e a sonolência ao acordar as queixas mais frequentes, quando consideradas em relação à sua gravidade. Estes resultados, são consistentes com a literatura internacional (Archobold e cols., 2002; Blader \& Gallagher, 2001; Manni e cols., 1997), que tem persistentemente alertado para as implicações destes problemas ao nível do bem-estar das crianças. Efectivamente alguns estudos longitudinais têm demonstrado que os problemas de sono, não raras vezes menosprezados na infância, podem evoluir e dar origem a graves problemas médico-psiquiátricos na idade adulta (Kerr \& Jowett, 1994; Stores, 1996; Wolfson \& Carskadon, 1998).

A maioria das crianças do nosso estudo refere dormir 9 ou mais horas, dado este que é consistente com dados de outros países europeus (Mantz \& Muzet, 1992; Ottaviano, Giannotti, Cortesi, Bruni \& Ottaviano, 1996). Porém, existe um considerável número de crianças que dormem menos de 5 horas por noite, resultado ao qual deverá ser dado uma maior ênfase em estudos posteriores e, também, ao nível da prática clínica. $\mathrm{Na}$ realidade, alterações ainda que pequenas, na hora de deitar ou de acordar das crianças, de modo a ampliar o tempo de sono, afectam consideravelmente funções neuro-comportamentais, melhorando o desempenho da criança ao nível do rendimento cognitivo e comportamental relacionado com processos de atenção, memória, concentração e rendimento académico (Sadeh, Raviv \& Gruber, 2000). Neste sentido, e na medida em que o instrumento utilizado apenas avaliava o número de horas de sono por noite, esta hipótese pode ser viável para explicar este sintoma que se destaca de entre muitos outros. Seria pertinente acrescentar em estudos semelhantes, a título posterior, a avaliação relativamente à hora de deitar, de forma a validar ou não a hipótese levantada.

O sono é sensível a influências culturais e psicossociais como a idade e o sexo da criança, a educação dos pais, trabalhos de casa, actividades extracurriculares, jogos de computador e desportos, que muitas vezes impõem um padrão de sono irregular, insatisfatório em ambientes e condições inadequadas. Muitas vezes são algumas destas variáveis, nomeadamente actividades escolares ou lúdicas que as crianças realizam antes de deitar que adiam a hora a que estas adormecem (Tsai \& Li, 2004). Os resultados deste estudo revelaram algumas diferenças geográficas e também de género relativas a problemas de sono, as quais deverão ser tidas em conta e exploradas em futuras investigações. Porém, seria prematuro afirmar a real existência destas diferenças em termos das variáveis consideradas, uma vez que os dados deste estudo não são suficientes para extrapolar considerações suficientemente claras a esse respeito.

Os resultados revelam ainda que, à medida que as crianças crescem, diminuem os sintomas relativos às insónias nocturnas e a sonolência ao acordar. Verificou-se também que as crianças com 8 e com 9 anos são as que apresentam mais problemas de sono e perturbação de sono. Sabe-se que a arquitectura do sono se altera em função da idade (Roffwarg, Muzio \& Dement, 1996). Deste modo, por volta dos seis anos a proporção de sono REM começa a diminuir, até estabilizar numa proporção de 20\% por noite (Anders, Sadeh \& Appareddy, 1995). A duração do período de sono diminui durante a idade escolar (Tynjälä, Villberg \& Kannas, 2002). Num estudo baseado na actigrafia, as crianças entre os 6 aos 12 anos referiam dormir em média, aproximadamente 9 horas por noite; porém na realidade este intervalo variava entre as $7 \mathrm{~h} 20 \mathrm{mn}$ e as $10 \mathrm{~h} 12 \mathrm{mn}$ de sono por noite (Aronen, Paavonen, Soininen \& Fjällberg, 2001). Estes resultados são correspondentes com as medidas de auto-relato na mesma faixa etária, e em que aos 9 anos as crianças referem dormir 9h14mn e aos 11 anos 8h51mn (Tynjälä e cols., 2002).

A utilização de medidas de auto-relato tem sido largamente utilizada em estudos epidemiológicos, embora delas possam advir problemas de validade e fidelidade. Para colmatar esta limitação são usados questionários estandardizados. O questionário usado neste estudo, demonstrou uma elevada taxa de participação (91\%), o que pode ser perspectivado como indicador da facilidade de compreensão desta medida subjectiva de avaliação de crianças em idade escolar. Porém, os dados poderão estar inflacionados por conterem falsos positivos e, por isso, seria desejável em estudos futuros recorrer a uma entrevista e/ou avaliações objectivas (isto é, actigrafia) para confirmar as respostas dadas ao questionário. No entanto, a discrepância entre medidas objectivas e subjectivas em crianças desta faixa etária têm sido pouco expressivas (Mantz \& Muzet, 1992; Ottaviano e cols., 1996).

Finalmente, este estudo pode contribuir para a existência de um conjunto de dados sobre o sono das crianças portuguesas em idade escolar, útil aos técnicos que se dedicam à intervenção/tratamento deste tipo de patologias na criança. Outra contribuição prende-se com um alertar, quer para os pais quer para os professores, sobre a influência que alterações nos padrões de sono das crianças podem, efectivamente, ter no bem-estar psicológico e rendimento cognitivo destas, nomeada- 
mente no nível do seu percurso escolar. Estando na posse destes dados, é possível então que sejam tomadas medidas para salvaguardar a higiene de sono nas crianças, bem como promover o ajustamento ao ritmo escolar.

Neste sentido, a prevenção, diagnóstico e tratamento das perturbações do sono está a tornar-se cada vez mais uma questão multidisciplinar. Muitas perturbações de sono estão associadas a comportamentos inadequados, que podem estar na sua origem, precipitação e/ou manutenção (Whitney, Enright, Newman, Bonekat, Foley \& Quan; 1998); mas que podem no entanto, ser revertidas quando os sintomas são identificados precocemente. A prevenção, torna-se um aspecto especialmente importante, quando a investigação demonstra que os problemas de sono, problemas sociais, comportamentais e de aprendizagem, com alguma probabilidade, podem predispor as crianças a perturbações de sono muito específicas, como a insônia, entre outras, na idade adulta. Deste modo, uma maior consciencialização por parte da comunidade, principalmente pais e agentes educativos, face a esta problemática poderá ajudar a identificar precocemente os sintomas e a colmatar consequências nefastas não só em nível escolar, como também e mais importante ainda no nível do bem-estar das crianças, pois são relações de carácter bidirecional.

\section{Referências}

Anders, T. F., Sadeh, A. \& Appareddy, V. (1995). Normal sleep in neonates and children. Em R. Ferber; M. Kryger (Orgs.). Principles and practice of sleep medicine in the child (pp. 7-18). Philadelphia, WB: Saunders.

Archbold, K. H., Pituch K. J., Panahi P. \& Chervin R. D. (2002). Symptoms of sleep disturbances among children at two general paediatrics clinics. Journal of Pediatric, 140, 97-102.

Aronen. E. T., Paavonen, E. J., Soininen, M. \& Fjällberg, M. (2001). Associations of age and gender with activity and sleep. Acta Paediatrica, 90, 222- 4.

Blader, J. C. \& Gallagher, R. (2001). Consultation to administrators. Child and Adolescent Psychiatric Clinics of North America, 10, 185-197.

Blader, J. C., Koplewicz, H. S., Abikoff, H. \& Foley, E. (1997). Sleep problems of elementary school children. A community survey. Archives of Paediatric Adolescent Medicine, 151, 473-80.

Borges, B., Azevedo, M., Fonseca, P., Torres, P. \& Costa, F. M. (2000). Hábitos de sono. Saúde Infantil, 22(3), 61-68.

Chervin, R. D., Rillon, J. E., Basseti, C., Ganoczy, D. A. \& Pituch, K. J. (1997). Symptoms of sleep disorders, inattention and hyperactivity in children. Sleep, 20, 11851192.

Clemente, V. O., Silva, C., Ferreira, A. M., César, H. \& Azevedo, M. H. (1997). Terapia cognitivo-comportamental na insónia: uma abordagem eficaz mas... esquecida. Revista Portuguesa de Clínica Geral, 14(1), 49-58.

Dahl, R. E. (1989). The development and disorders of sleep. Advances in Paediatrics, 45, 73-89.

Diest, R. van (1990). Subjective sleep characteristics as coronary risk factors, their association with type A behaviour and vital exhaustion. Joumal of Psychosomatic Researcher, 34(4), 415-426.

Kahn, A., Merckt, C., Rebuffat, E., Mozin, M. J., Sottiaux, M., Blum, D. \& Hennart, P. (1989). Sleep problems in healthy preadolescents. Pediatrics, 84, 542-546.

Kerr, S. \& Jowett, S. (1994). Sleep problems in pre-school children: A review of the literature. Child Care Health Development, 20, 379-391.

Klackenberg, G. (1982). Sleep behaviour studied longitudinally. Data from 4-16 years on duration, nightawakeningand bed-sharing. Acta Paediatrica Scandinavica, 71, 501-6.

Klein, J. M., Fernandes, C. \& Gonçalves, A. (2005). Epidemiological study of sleep disorders in school-aged childrens of Portuguese schools. (Abstract). Psychophysiology, 42 (Suplement), 74.

Klein, J. M., Silva, C. F. \& Gonçalves, A. (no prelo) Assessing sleep-wake complaints in school-aged children: Portuguese properties and norms of the SWEL. Psiquiatria Clinica.

Manni, R., Ratti, M. T., Marchioni, E., Castelnovo, G., Murelli, R., Sartori, I., Galimberti, C. A. \& Tartara, A. (1997). Poor sleep in adolescents: a study of 86917 -yearold Italian secondary school students. Journal of Sleep Research, 6, 44-49.

Mantz, J. \& Muzet, A. (1992). School physician and rhythms of schoolchildren's life. Annals of Paediatrics, 39(5), 317-21.

Mindell, J. A., Moline, M. L., Zendell, S. M., Brown, Z. M. \& Frey, J. M. (1994). Paediatricians and sleep disorders: training and practice. Paediatrics, 94, 194-200.

Mindell, J., Boaz, A., Joffe, M., Curtis, S. \& Birley, M. (2004). Evidence based public health policy and practice: Enhancing the evidence base for health impact assessment. Journal of Epidemiology Community Health, 58, 546-55.

Ohayon, M. M. (2002). Epidemiology of insomnia: What we know and what we still need to learn. Sleep Medicine, 2(6), 97-111. 
Ottaviano, S., Giannotti, F., Cortesi, F., Bruni, O. \& Ottaviano, C. (1996). Sleep characteristics in healthy children from birth to 6 years of age in the urban area of Rome. Sleep, 19(1), 1-3.

Pollock, J. L. (1994). Night-waking at five years of age: Predictors and prognosis. Journal of Child Psychology and Psychiatry, 35(4), 699-708.

Roffwarg, H. P., Muzio, J. N. \& Dement, W. C. (1966). Ontogenetic development of the human sleep-dream cycle. Science, 152, 604-19.

Rona, K. J., Li, L., Gulliford, M. C. \& Chinun, S. (1998). Disturbed sleep: effects of socio-cultural factors and illness. Archives of Disorders in Childhood, 78, 28-51.

Sadeh, A., Raviv, A. \& Gruber, R. (2000). Sleep patterns and sleep disruptions in school-age children. Developmental Psychology, 36(3), 291-301.

Simonds, J. F. \& Parraga, H. (1984). Prevalence of sleep disorders and sleep behaviors in children and adolescents. Journal of the American Academy of Child Psychiatry, 21, 383-388.

SPSS for Windows, Release 12.0.0 (2004). Chicago: SPSS Incorporated.

Stores, G. (1996). Practioner review: Assessment and treatment of sleep disorders in children and adolescents. Journal of Child Psychology and Psychiatry, 37, 907-925.

Terman, L. M. \& Hocking, A. (1913). The sleep of school children, its distribution according to age, and its relation to physical and mental efficiency. Journal of Educational Psychology, 4, 138-147.

Tsai, L-L \& Li, S-P. (2004). Sleep patterns in college students: Gender and grade differences. Journal of Psychosomatic Researcher, 56, 231-237.

Tynjälä, J., Villberg, J. \& Kannas, L. (2002). Nuorten nukkumistottumukset ja väsyneisyys vuosina 1984-98. Suomen Lääkärilebti, 57, 2993-98.

Whitney, C. W., Enright, P. L., Newman, A. B., Bonekat, W., Foley, D. \& Quan, S. F. (1998). Correlates of daytime sleepiness in 4578 elderly persons: The cardiovascular health study. Sleep, 21(1), 27-36.

Wiggs, L. \& Stores, G. (2001). Sleepiness. Em G. Stores \& L. Wiggs (Orgs.). Sleep disturbance in cbildren and adolescents with disorders of development: Its significance and management. Clinics in Developmental Medicine (Vol. 155, pp. 24-29). UK: Cambridge University Press.

Wolfson, A. \& Carskadon, M. (1998). Sleep schedules and daytime functioning in adolescents. Child Development, 69, 875-887.

Zuckerman, B., Stevenson, J. \& Bailey, V. (1987). Sleep problems in early childhood: continuities, predictive factors, and behavioural correlates. Paediatrics, 80, 664-71.

Recebido em maio de 2007 Reformulado em agosto de 2007 Aprovado em setembro de 2007

Sobre os autores:

John M. Klein é doutor em Psicologia Clínica e atualmente professor na Universidade de Trier (Alemanha). Tem publicado e apresentado vários trabalhos nas seguintes áreas de interesse: Psicopatologia, Processos e Resultados Terapêuticos, Avaliação Psicológica e Neurociências.

Alda Gonçalves é licenciada em Psicologia pela Universidade do Minho e mestranda em Psicologia Clínica, na mesma universidade. Nos últimos anos, tem-se dedicado a estudar as perturbações do comportamento alimentar e as perturbações de sono. 